\title{
Heart Rate Variability Analysis Assessment for Asthma Control Stratification
}

\author{
Javier Milagro ${ }^{1,2}$, Lorena Soto ${ }^{3}$, Jordi Giner ${ }^{3}$, Carolina Varon ${ }^{4}$ \\ Pablo Laguna ${ }^{1,2}$, Vicente Plaza ${ }^{3}$, Eduardo Gil ${ }^{1,2}$, Raquel Bailón ${ }^{1,2}$ \\ ${ }^{1}$ BSICoS Group, I3A, IIS Aragón, University of Zaragoza, Zaragoza, Spain \\ ${ }^{2}$ CIBER - Biengineering, Biomaterials and Nanomedicine (CIBER-BBN), Madrid, Spain \\ ${ }^{3}$ Pneumology and Allergy Department, Santa Creu i Sant Pau Hospital, Barcelona, Spain \\ ${ }^{4}$ ESAT, STADIUS, KU Leuven, Leuven, Belgium
}

\begin{abstract}
Autonomic nervous system (ANS) has been suggested to play a major role in the pathogenesis of asthma. This hypothesis has motivated large research, revealing a reduced modulation of the heart rate in subjects with uncontrolled asthma, when compared to asthmatics with controlled symptomatology. In this work, we assessed ANS activity through heart rate variability analysis in a group of asthmatics classified attending to the control of their symptoms. This information was later used for training a logistic regression classifier aiming at differentiating between the levels of control in asthmatic patients. The accuracy of the classifier improved when including ANS information (71.77\%, versus $64.73 \%$ when only clinical parameters were considered), suggesting that ANS assessment could contribute to better non-invasive asthma monitoring.
\end{abstract}

\section{Introduction}

Asthma is a heterogeneous chronic respiratory disorder whose prevalence has been increasing since the second half of last century [1]. It is characterized by a variable expiratory airflow limitation and bronchial hyperresponsiveness. Despite asthma is usually accompanied by chronic airway inflammation, there is controversy regarding the role of pro-inflammatory substances and inflammatory cells in the bronchial hyper-responsiveness. Since upper airway constriction is governed by vagal activity, the role of the autonomic nervous system (ANS) in the pathogenesis of asthma has attracted research interest in recent years, with several studies suggesting that sympathovagal activity might be altered in asthmatics $[2,3]$.

Whereas the physiological underlying origin of asthma is not completely understood, its diagnosis and monitoring in adults is performed through a well-established clinical routine, consisting in the measurement of airway function using spirometric tests and in the assessment of inflammatory markers such as the levels of exhaled nitric oxide (FeNO) or immunoglobulin E (IgE). Also self-applied questionnaires are employed for evaluating whether the symptomatology remains controlled or not, although these surveys remain rather subjective. Therefore, non-invasive ANS assessment techniques, such as heart rate variability (HRV) analysis have been considered, suggesting a decreased cardiac autonomic modulation in uncontrolled when compared with controlled asthma [4]. Hence, it is possible that ANS assessment through HRV analysis may add some clinical value for the automatic stratification of asthmatic subjects. Departing from this hypothesis, we evaluated the HRV of a group of asthmatic adults classified attending to their asthma control level, and further used this information for improving the stratification of the analyzed subjects in controlled and uncontrolled asthma.

\section{Materials and Methods}

\subsection{Database}

The dataset in the study consists of recordings from 30 asthmatic volunteers classified attending to their level of control of the asthmatic symptoms (19 with controlled asthma, CA, and 11 with uncontrolled asthma, UA). Classification was performed according to the asthma control test (ACT) score (UA if ACT $\leq 19$, CA otherwise) [5]. All of them were patients of the Santa Creu i Sant Pau Hospital in Barcelona (Spain), and provided a written informed consent in agreement with the Declaration of Helsinki before being included in this study. The volunteers underwent a biosignals acquisition protocol: they remained sat and without talking for 10 minutes, during which multilead ECG and respiratory effort (using a respiratory belt) signals were acquired, at sampling rates of 1000 and 256 $\mathrm{Hz}$ respectively. Afterwards, they performed a spirometric 
Table 1. Demographics and clinical features of the two groups. Non-categorical parameters are displayed as median (interquartile range). Statistical significant differences among groups $(\mathrm{p}<0.05)$ are indicated with *. $(\mathrm{N}$ : number of subjects, BMI: body mass index.)

\begin{tabular}{lcc}
\hline \hline & Controlled Asthma & Uncontrolled Asthma \\
\hline Demographics: & 19 & 11 \\
$\bullet$ N (\#) & $50.00(19.00)$ & $49.00(20.50)$ \\
- Age (years) & $11 / 8$ & $2 / 9^{*}$ \\
- Gender (Male/Female) & $26.40(3.90)$ & $30.00(8.25)^{*}$ \\
• BMI (kg/m $\left.{ }^{2}\right)$ & & \\
\hline Clinical features: & $16 / 3$ & $8 / 3$ \\
- Atopy (Yes/No) & $73.00(10.50)$ & $56.00(23.25)$ \\
- FEV $/$ FVC $(\%)$ & $27.00(13.75)$ & $41.00(65.63)$ \\
$\bullet$ FeNO (ppb) & $7 / 12$ & $6 / 5$ \\
- Periph Eos (Yes/No) & $131.00(149.50)$ & $204.00(449.93)$ \\
- IgE (UI/ml) & $4 / 15$ & $3 / 8$ \\
- Inflam (Yes/No) & & \\
\hline \hline
\end{tabular}

test in which their forced expiratory volume in one second relative to their forced vital capacity $\left(\mathrm{FEV}_{1} / \mathrm{FVC}\right)$ was obtained. Also their level of FeNO, blood IgE, peripheral eosinophils count and induced sputum inflammatory cells count were assessed. The two latter measurements were used by the clinicians to establish whether the subjects presented peripheral eosinophilia (periph eos) or airway inflammation (inflam). The demographics and measured clinical parameters of the subjects are summarized in Table 1. None of them presented cardiac, neurological or endocrine disease, nor other obstructive disease different from asthma at the time of the study.

\subsection{Signal Preprocessing}

Baseline wander was removed from the ECGs, and respiratory signals were filtered with a $3^{\text {rd }}$-order Butterworth band-pass filter (0.05-1 Hz cut-off frequencies). Then, $\mathrm{R}$ peak detection was performed using a wavelet-based approach [6], and ectopic beats $(0.13 \%$ of the total number of beats) were detected and discarded from the analysis using the method presented in [7]. The respiratory signals were downsampled at $4 \mathrm{~Hz}$, and used for estimating the instantaneous respiratory rate, as proposed in [8].

\subsection{Heart Rate Variability Analysis}

The modulating signal, $m(t)$, which carries information from the autonomic modulation of the heart rate (HR) was estimated using the time-varying integral pulse frequency modulation model [9], which accounts for the presence of ectopic beats. This model allows to obtain the instantaneous HR signal, $d_{\mathrm{HR}}(t)$, from the beat time occurrences. Then, $d_{\mathrm{HR}}(t)$ was low-pass filtered to calculate a timevarying mean $\mathrm{HR}, d_{\mathrm{HRM}}(t)$, and $m(t)$ was obtained as:

$$
m(t)=\frac{\left(d_{\mathrm{HR}}(t)-d_{\mathrm{HRM}}(t)\right)}{d_{\mathrm{HRM}}(t)} .
$$

Finally, $m(t)$ was sampled at $4 \mathrm{~Hz}$ and, for simplicity, it is referred to as $\mathbf{m}$ (vector notation) hereon.

A preliminary analysis revealed the existence of respiratory rates lower than or very close to $0.15 \mathrm{~Hz}$, frequency considered the limit between low-frequency (LF) and highfrequency (HF) bands $([0.04,0.15] \mathrm{Hz}$ and $[0.15,0.4] \mathrm{Hz}$ respectively) in HRV studies, in the $13 \%$ of the subjects. Since the power content of the HF band has been traditionally related with respiratory modulation of the HR, the interpretation of the frequency components laying within it when respiratory influence is shifted towards the LF band remains an open debate, and traditional frequency-domain HRV analysis is compromised [10]. For this reason, in this work we addressed the HRV analysis using an orthogonal subspace projection (OSP) decomposition approach [11]. Essentially, respiratory information is removed from $\mathbf{m}$ using a projection matrix, $\mathbf{P}$, which is defined as:

$$
\mathbf{P}=\mathbf{X}\left(\mathbf{X}^{\mathbf{T}} \mathbf{X}\right)^{-\mathbf{1}} \mathbf{X}^{\mathbf{T}}
$$

where $\mathbf{X}$ is constructed using delayed versions of the respiratory effort signal up to 2 seconds [11]. Afterwards, the respiratory-related component of $\mathbf{m}$ can be obtained as:

$$
\mathbf{m}_{\text {respir }}=\mathbf{P m},
$$

and the residual component, i.e., the non-respiratory related component, was calculated as:

$$
\mathbf{m}_{\text {resid }}=(\mathbf{I}-\mathbf{P}) \mathbf{m}=\mathbf{m}-\mathbf{m}_{\text {respir }},
$$

where $\mathbf{I}$ is the identity matrix. Afterwards, the spectra of both components were obtained using the Welch's periodogram (50 s windows, 50\% overlap) approach (an example of the resulting spectra is displayed in Fig. 1). Whereas non-respiratory related power, $\mathrm{P}_{\text {resid }}^{\mathrm{LF}}$, was computed as the power content of $\mathbf{m}_{\text {resid }}$ within the $\mathrm{LF} \mathrm{Hz}$ band, the power of the respiration-related component, $\mathrm{P}_{\text {respir }}$, was obtained as the power of $\mathbf{m}_{\text {respir }}$ in the $[0.04, \overline{\mathrm{HR}} / 2] \mathrm{Hz}$ band (being $\overline{\mathrm{HR}}$ the mean $\mathrm{HR}$ in $\mathrm{Hz}$ ). Also the ratio $\mathrm{R}_{\mathrm{OSP}}=\mathrm{P}_{\text {resid }}^{\mathrm{LF}} / \mathrm{P}_{\text {respir }}$ was computed as an alternative sympathovagal balance measurement [11]. Finally, the total power (TP) was calculated as the sum of the classical LF and HF powers. The described indexes were calculated from five-minute windows of $\mathbf{m}$, with four-minute overlap.

\subsection{Statistical Analysis}

Subjects were characterized by the median of their HRV parameters. Normality of the data was rejected using a Kolmogorov-Smirnov test, so differences among groups were assessed using a Wilcoxon rank-sum test, setting the significance threshold to 0.05 . 


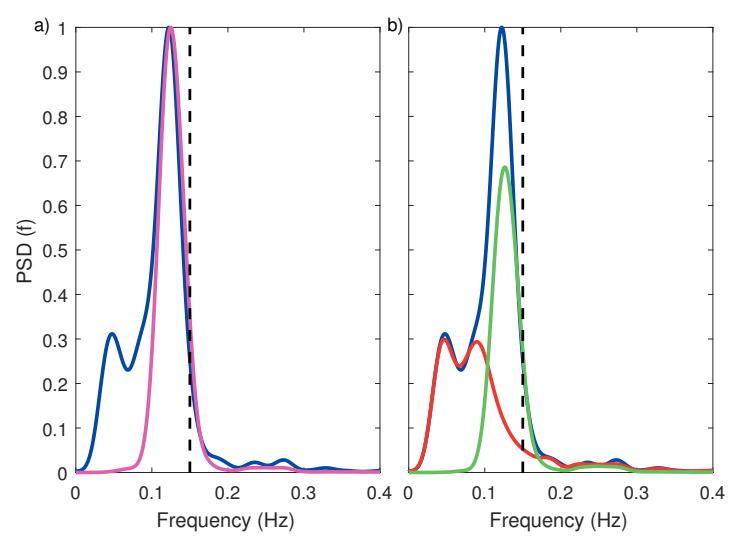

Figure 1. Normalized power spectral density of the modulating signal (blue) and the respiratory effort (pink) in a five-minute segment are displayed in a). Since the respiratory activity lays below $0.15 \mathrm{~Hz}$ (black dashed line), in b) orthogonal subspace projection was applied to separate the respiratory-related (green) and -unrelated (red) components of the modulating signal.

\subsection{Logistic Regression Classifier}

A logistic regression (LR) classifier was used for stratifying the subjects in CA and UA. Sequential feature selection was applied to all the clinical parameters by maximizing the accuracy. Then, the two best performing features were combined with the introduced HRV measurements. The classifier was trained using the leave-one-out technique combined with bootstrapping in order to avoid overfitting [12], following the scheme in [13] (100 bootstrapped training sets were used for each subject). Then, the accuracy, sensitivity, specificity and F1 score were calculated as the mean values of all the constructed classifiers.

\section{Results}

Differences in the HRV parameters among groups and the performance of the classifier for several sets of features are summarized in Tables 2 and 3 respectively. Attending to HRV analysis, decreased TP, $\mathrm{P}_{\text {resid }}^{\mathrm{LF}}$ and $\mathrm{P}_{\text {respir }}$ were found in the UA group, although no difference in $\mathrm{R}_{\mathrm{OSP}}$ was assessed.

On the other hand, $\mathrm{FEV}_{1} / \mathrm{FVC}$ and IgE levels were selected as the clinical features that achieved the best accuracy $(64.73 \%)$, closely followed by the FeNO (although adding the latter to the classifier did not improve the accuracy, so it is not considered hereon). The use of $\mathrm{P}_{\text {resid }}^{\mathrm{LF}}$ and $\mathrm{R}_{\mathrm{OSP}}$ resulted in a similar performance than the clinical features, whereas combining any of them with $\mathrm{FEV}_{1} / \mathrm{FVC}$ and $\operatorname{IgE}$ improved the classification accuracy. Moreover, adding both HRV features simultaneously resulted in the best performance, with an accuracy of $71.77 \%$ and an increase in sensitivity and specificity of more than a $5 \%$ and
Table 2. Median (interquartile range) of the heart rate variability parameters for each group. Statistical significant differences among groups $(\mathrm{p}<0.05)$ are indicated with *

\begin{tabular}{lcc}
\hline \hline & Controlled Asthma & Uncontrolled Asthma \\
\hline HR (beats per min) & $73.98(22.48)$ & $77.79(7.86)$ \\
TP $\left(\right.$ a.u. $\left.\times 10^{3}\right)$ & $13.65(10.16)$ & $4.85(3.13)^{*}$ \\
$\mathrm{P}_{\text {resid }}^{\mathrm{L}}\left(\right.$ a.u. $\left.\times 10^{3}\right)$ & $5.67(6.18)$ & $2.02(1.67)^{*}$ \\
$\mathrm{P}_{\text {respir }}\left(\right.$ a.u. $\left.\times 10^{3}\right)$ & $2.66(3.89)$ & $0.85(1.43)^{*}$ \\
$\mathrm{R}_{\text {oSP }}$ (n.u. $)$ & $2.38(2.20)$ & $2.19(4.78)$ \\
\hline \hline
\end{tabular}

Table 3. Mean accuracy (Acc), sensibility (Sens), specificity (Spec) and F1 score of the classifier using different sets of features (displayed between brackets).

\begin{tabular}{lcccc}
\hline \hline & Acc (\%) & Sens (\%) & Spec (\%) & $\mathrm{F} 1$ \\
\hline$\left\{\mathrm{FEV}_{1} / \mathrm{FVC}, \mathrm{IgE}\right\}$ & 64.73 & 79.00 & 40.09 & 0.74 \\
$\left\{\mathrm{P}_{\text {resid }}^{\mathrm{LF}}, \mathrm{R}_{\mathrm{OSP}}\right\}$ & 64.60 & 82.84 & 33.09 & 0.75 \\
$\left\{\mathrm{FEV}_{1} / \mathrm{FVC}, \mathrm{IgE}, \mathrm{P}_{\text {resid }}^{\mathrm{LF}}\right\}$ & 68.33 & 79.58 & 48.91 & 0.76 \\
$\left\{\mathrm{FEV}_{1} / \mathrm{FVC}, \mathrm{IgE}, \mathrm{R}_{\text {OSP }}\right\}$ & 67.03 & 83.00 & 39.45 & 0.76 \\
$\left\{\mathrm{FEV}_{1} / \mathrm{FVC}, \mathrm{P}_{\text {resid }}^{\mathrm{LF}}, \mathrm{R}_{\text {OSP }}\right\}$ & 70.37 & 85.37 & 44.45 & 0.79 \\
$\left\{\mathrm{FEV}_{1} / \mathrm{FVC}, \mathrm{IgE}, \mathrm{P}_{\text {resid }}^{\mathrm{LF}}, \mathrm{R}_{\text {OSP }}\right\}$ & $\mathbf{7 1 . 7 7}$ & $\mathbf{8 4 . 3 7}$ & $\mathbf{5 0 . 0 0}$ & $\mathbf{0 . 7 9}$ \\
\hline \hline
\end{tabular}

almost a $10 \%$ respectively, when compared with the classification using only clinical features. Finally, replacing the $\operatorname{IgE}$ by $\mathrm{P}_{\text {resid }}^{\mathrm{LF}}$ and $\mathrm{R}_{\mathrm{OSP}}$ also resulted in an improved performance with respect to the classification using $\mathrm{FEV}_{1} / \mathrm{FVC}$ and IgE. Slightly reduced performance was achieved when considering TP instead of $\mathrm{P}_{\text {resid }}^{\mathrm{LF}}$.

\section{Discussion}

In this work, the inclusion of ANS activity information for the improvement of automatic asthmatic subjects classification has been proposed. ANS assessment was accomplished using HRV analysis, which remains highly non-invasive. Since a preliminary study revealed a generally low respiratory rate that might compromise the interpretation of a traditional frequency-domain HRV analysis, we proposed the use of an OSP decomposition of the HR modulating signal, which allows to uncouple the respiratory linear-related and -unrelated components of HRV [11]. As displayed in Table 2, the frequency-domain analysis of these components revealed a decreased power in both of them in the UA group, thus suggesting a reduced autonomic modulation of HR in subjects with a worse asthma control. Whereas previous studies revealed an increased vagal response to autonomic tests in asthmatics than in controls [2] that appears to be related with asthma severity [3], Lutfi et al. reported a global decreased HRV in awake adults with uncontrolled asthma when compared with controlled asthmatics [4], in concordance with this study.

Increased body mass index and a larger relative number of females were assessed in the UA group. Whereas obesity has been related with decreased HRV in previ- 
ous studies [14], females usually present decreased sympathetic tone and increased vagal tone than males [15], which could compromise the interpretation of the aforementioned results. However, the body mass index was uncorrelated with all the HRV measurements and the respiratory-related component of HRV was lower instead of higher in UA, suggesting that the differences in ANS activity between groups may be due to other causes than obesity or gender.

Since the monitoring of an individual asthmatic status can not be performed in a continuous-time manner and might require to visit the hospital and some invasive measurement such as blood tests, we postulated that the inclusion of ANS information could result in a better stratification of the subjects attending to the control of the disease. In this way, we employed a LR classifier which was trained with different sets of features in order to compare their classification performance. Unfortunately, the small sample size did not allow to have separated test and train sets. Applying a sequential feature selection to all the available clinical features raised $\mathrm{FEV}_{1} / \mathrm{FVC}$ and $\mathrm{IgE}$ as those that maximized the accuracy of the classifier. As displayed in Table 3, including either $\mathrm{P}_{\text {resid }}$ or $\mathrm{R}_{\mathrm{OSP}}$ slightly increased the classification performance. Moreover, including both features simultaneously resulted in the best performance, with a $7 \%$ increase in the classification accuracy with respect to the case when only clinical features were employed, although it could be related with the use of a higher number of features. On the other hand, if the HRV features were used to substitute $\operatorname{IgE}$, the accuracy also raised almost a $5 \%$. Therefore, the inclusion of ANS information might be a suitable complement for the clinical practice, which could improve the self-monitoring in asthmatic subjects.

\section{Conclusion}

HRV analysis revealed a decreased autonomic modulation of the HR in uncontrolled asthmatics when compared with controlled asthmatics, in concordance with previous works. The inclusion of ANS information resulted in a better stratification of the subjects than using only clinical features, which encourages further research in the field of non-invasive asthma monitoring.

\section{Acknowledgements}

This work was supported by grant BES-2015-073694 and project RTI2018-097723-B-I00 from Ministerio de Economía y Competitividad, by Gobierno de Aragón (Reference Group BSICoS T39-17R) cofunded by FEDER 2014-2020 "Building Europe from Aragon" and by CIBER-BBN through Instituto de Salud Carlos III. The computation was performed by the ICTS NANBIOSIS, specifically by the High Performance Computing Unit of CIBER-BBN at University of Zaragoza. C. Varon is a postdoctoral fellow of the Research Foundation-Flanders.

\section{References}

[1] Lundbäck B, et al. Is asthma prevalence still increasing? Expert review of respiratory medicine 2016;10(1):39-51.

[2] Shah PK, et al. Clinical dysautonomia in patients with bronchial asthma: study with seven autonomic function tests. Chest 1990;98(6):1408-1413.

[3] Emin O, et al. Autonomic nervous system dysfunction and their relationship with disease severity in children with atopic asthma. Respir Physiol Neurobiol 2012;183(3):206210.

[4] Lutfi MF. Patterns of heart rate variability and cardiac autonomic modulations in controlled and uncontrolled asthmatic patients. BMC Pulm Med 2015;15(1):119.

[5] Vega J, et al. Validation of the spanish version of the asthma control test (ACT). J Asthma 2007;44(10):867-872.

[6] Martínez JP, et al. A wavelet-based ECG delineator: evaluation on standard databases. IEEE Trans Biomed Eng 2004; 51(4):570-581.

[7] Mateo J, Laguna P. Analysis of heart rate variability in the presence of ectopic beats using the heart timing signal. IEEE Trans Biomed Eng 2003;50(3):334-343.

[8] Bailón R, et al. A robust method for ECG-based estimation of the respiratory frequency during stress testing. IEEE Trans Biomed Eng 2006;53(7):1273-1285.

[9] Bailón R, et al. The integral pulse frequency modulation model with time-varying threshold: application to heart rate variability analysis during exercise stress testing. IEEE Trans Biomed Eng 2011;58(3):642-652.

[10] Bernardi L, et al. Effects of controlled breathing, mental activity and mental stress with or without verbalization on heart rate variability. J Am Coll Cardiol 2000;35(6):14621469.

[11] Varon C, et al. Unconstrained estimation of HRV indices after removing respiratory influences from heart rate. IEEE J Biomed Health Inform 2018 DOI 10.1109/JBHI.2018.2884644.

[12] Efron B, Tibshirani RJ. An introduction to the bootstrap. CRC press, 1994.

[13] Bolea J, et al. Pulse rate and transit time analysis to predict hypotension events after spinal anesthesia during programmed cesarean labor. Ann Biomed Eng 2017; 45(9):2253-2263.

[14] Thayer JF, et al. The relationship of autonomic imbalance, heart rate variability and cardiovascular disease risk factors. Int J Cardiol 2010;141(2):122-131.

[15] Antelmi I, et al. Influence of age, gender, body mass index, and functional capacity on heart rate variability in a cohort of subjects without heart disease. Am J Cardiol 2004; 93(3):381-385.

Address for correspondence:

Javier Milagro

Dep. Ingeniería Electrónica y Comunicaciones. Universidad de Zaragoza, C/ María de Luna 1, L.3.07, 50018 Zaragoza, Spain milagro@unizar.es 